\title{
Educação permanente em saúde no contexto de uma unidade de saúde da família no município do Rio de Janeiro, Brasil
}

\author{
Permanent health education in the context of a family health unit in the municipality of Rio \\ de Janeiro, Brazil \\ Educación de salud permanente en el contexto de una unidad de salud familiar en la \\ ciudad de Río de Janeiro, Brasil
}

Josinei Feijó da Silva ${ }^{1 *}$, Carlos Otávio Fiuza Moreira², Tarciso Feijó da Silva ${ }^{3}$, Vagner Ferreira do Nascimento ${ }^{4}$, Fagner Luiz Lemes Rojas ${ }^{4}$, Rodrigo Ayres de Souza ${ }^{5}$.

\section{RESUMO}

Objetivo: Analisar a percepção dos profissionais sobre as práticas de Educação Permanente em Saúde, considerando a relação entre a prática profissional e a produção de saberes. Métodos: Pesquisa de campo, de natureza qualitativa realizada a partir de grupos focais com profissionais da Estratégia de Saúde da Família no Município do Rio de Janeiro. Resultados: Foram identificados dois diferentes modos de compreender e utilizar a Educação Permanente em Saúde, primeiro, como espaço de produção dos saberes para a circulação do conhecimento e, segundo, como uma forma de reflexão pelos profissionais de saúde da sua prática e das ações em saúde que realizam. Observou-se, ainda, fragilização na formação técnica do agente comunitário de saúde, com possíveis efeitos no desempenho das equipes de saúde da família e no cuidado em saúde. Conclusão: Embora existam políticas indutoras que orientam a Educação Permanente em Saúde, a efetividade do seu potencial para produção de saberes na prática dos profissionais que atuam na Saúde da Família permanece como incipiente e constitui-se num desafio para o Sistema Único de Saúde.

Palavras-chave: Educação em saúde, Educação continuada, Saúde da família.

\section{ABSTRACT}

Objective: To analyze the perception of professionals about the practices of Permanent Education in Health, considering the relationship between professional practice and the production of knowledge. Methods: Qualitative field research carried out from focus groups with professionals from the Family Health Strategy in the city of Rio de Janeiro. Results: Two different ways of understanding and using Permanent Education in Health were identified, first, as a space for the production of knowledge for the circulation of knowledge and, second, as a way for health professionals to reflect on their practice and actions in health they perform. There was also a weakness in the technical training of the community health agent, with possible effects on the performance of family health teams and health care. Conclusion: Although there are inductive policies that guide Permanent Health Education, the effectiveness of its potential for producing knowledge in the practice of professionals working in Family Health remains incipient and constitutes a challenge for the Unified Health System.

Keywords: Health education, Continuing education, Family health.

\section{RESUMEN}

Objetivo: Analizar la percepción de los profesionales sobre las prácticas de Educación Permanente en Salud, considerando la relación entre la práctica profesional y la producción de conocimiento. Métodos: Investigación de campo, de carácter cualitativo, realizada desde grupos focales con profesionales de la Estrategia de Salud Familiar en la ciudad de Río de Janeiro. Resultados: Se identificaron dos formas diferentes de entender y utilizar la Educación Permanente en Salud, primero, como un espacio para la producción de conocimiento para la circulación del conocimiento y, en segundo lugar, como una forma de reflexión por parte de los profesionales de la salud de su práctica y acciones en salud que realizan. También hubo una debilidad en la

\footnotetext{
${ }^{1}$ Prefeitura Municipal de Cantagalo, Cantagalo - RJ. *E-mail: josineif.silva@yahoo.com.br

${ }^{2}$ Fundação Oswaldo Cruz, Rio de Janeiro - RJ.

3 Universidade do Estado do Rio de Janeiro (UERJ), Rio de Janeiro - RJ.

${ }^{4}$ Universidade do Estado de Mato Grosso (UNEMAT), Mato Grosso - MT.

${ }^{5}$ Prefeitura Municipal do Rio de Janeiro, Rio de Janeiro - RJ.
} 
capacitación técnica del agente de salud de la comunidad, con posibles efectos en el desempeño de los equipos de salud familiar y la atención médica. Conclusión: Aunque existen políticas inductivas que guían la Educación Permanente en Salud, la efectividad de su potencial para generar conocimiento en la práctica de los profesionales que trabajan en Salud Familiar sigue siendo incipiente y constituye un desafío para el Sistema Único de Salud.

Palabras clave: Educación para la salud, Educación continua, Salud familiar.

\section{INTRODUÇÃO}

O Ministério da Saúde, em 1994, criou o Programa de Saúde da Família (PSF), a partir das experiências ocorridas em países como Canadá, Cuba e Inglaterra e, também, em observância aos resultados positivos desenvolvidos pelo programa de agentes comunitários de saúde (PACS) no Brasil, principalmente no estado do Ceará (FARIA HP, et al., 2010).

O PSF, em 1998, tornou-se a principal estratégia para reorientação dos serviços de saúde até meados do início da década de 1990, em que o serviço de saúde ainda possuía forte centralização e ênfase na atenção médico-hospitalar, com visível falta de investimentos em iniciativas voltadas para prevenção e promoção em saúde. Essa reordenação da 'porta de entrada' ao SUS, em 2006, passou a denominar-se Estratégia de Saúde da Família (ESF) (NETO MMC, 2000).

Considerando a cobertura do Programa de Agentes Comunitários, no Brasil, observou-se que, até novembro de 2016, o PACS contava com 5.507 equipes implantadas e cobertura populacional estimada de 66,66\%, não havendo mudança significativa quando comparada ao ano de 2015 (66,63\%).

Com relação às equipes de Saúde da Família, a cobertura populacional estimada passou de $63,45 \%$, em 2015, para 63,62\%, em 2016, com 40.067 equipes implantadas nos 5.382 municípios da federação (BRASIL, 2016).

No Município do Rio de Janeiro a partir de 2009, houve uma ampliação das ESF, iniciativa vinculada ao plano estratégico da Prefeitura, intitulado: Programa Saúde Presente, que consistia na implantação de novas unidades de ESF nos territórios integrados de atenção à saúde, tendo como eixo principal a expansão da cobertura, privilegiando essa modalidade de unidades de saúde. Como resultado, desde a implantação do novo modelo, a cobertura de ESF no município do RJ passou de 3,5\%, em janeiro de 2009, para 56,8\%, em setembro de 2016 (SMSDC, 2015; BRASIL, 2016).

Em suas análises Rosa WAG e Labate RC (2005) apontam a ESF como uma proposta de mudança na lógica do trabalho em saúde na Atenção Primária. Segundo as autoras, trata-se de um modelo centrado na família, que busca modificar o anterior, centrado na cura de doenças e no hospital, possibilitando, assim, às equipes de saúde da família uma maior compreensão do processo saúde/doença da população e a identificação de necessidades de intervenção que vão além de práticas curativas.

O trabalho em saúde é um desafio para a integralidade e o fortalecimento do controle social no Sistema Único de Saúde (SUS) (BRASIL, 2004). A Política Nacional de Educação Permanente em Saúde (PNEPS) incentivou a criação de Pólos de Educação Permanente em Saúde (PEPS).

Como espaços de diálogo entre os atores do campo da saúde os PEPS para Ceccim RB (2005) permitem repensar o ensino, a gestão setorial, as práticas de atenção e o controle social (quadrilátero da formação). No que, foi utilizado, nos estudos de Rojas FFL (2018) através da Mandala da Educação Permanente em Saúde (EPS).

A EPS é uma proposta político-pedagógica que favorece, aos trabalhadores, um processo de ensino aprendizagem dentro do seu cotidiano laboral (GARCIA RM; BAPTISTA R, 2007), ou seja, é o desenvolvimento dos trabalhadores no cotidiano do trabalho que realizam, sendo centrada no próprio sujeito trabalhador que promove formas de aprender a aprender, a partir das situações laborais que vivencia (REZENDE MA, 2013).

A produção do conhecimento nos espaços formais, na qualificação do trabalho e nas instituições de ensino é quase sempre disciplinar e orientada pelo próprio processo de conhecimento (SOBRAL FAF, 2001). Desta 
forma, o foco da formação dos profissionais de saúde em referência à EPS converge para a necessidade da mudança na lógica das instituições de ensino, que deve incentivar à produção de conhecimento nos serviços envolvendo a análise das práticas dos profissionais de saúde, a atenção à saúde, a gestão setorial e a organização social (CECCIM RB, 2015).

Nesse contexto, a PNEPS considera que as práticas de EPS devem partir da problematização do processo de trabalho, em que são identificadas as necessidades de qualificação, garantindo a aplicação e a relevância dos conteúdos e das tecnologias estabelecidas (BRASIL, 2009).

Nesta ótica, a reflexão sobre o papel da EPS enquanto propulsora da produção de saberes a partir do trabalho desenvolvido pelos profissionais da ESF, assim como, a identificação de produção incipiente relacionada à temática neste ponto da rede de atenção em saúde construiu sentidos para desenvolvimento deste artigo que tem por objetivo analisar a percepção dos profissionais sobre as práticas de EPS, considerando a relação entre a prática profissional e a produção de saber.

\section{MÉTODOS}

Este artigo apresenta o resultado de uma pesquisa de campo, natureza qualitativa, que realizou um estudo descritivo e exploratório com 15 trabalhadores de saúde (médicos, enfermeiros e agentes comunitários de saúde) de sete ESF inseridas numa unidade de Atenção Primária na Zona Norte do Município do Rio de Janeiro, cujo cenário é um relevante espaço institucional para a prática de ensino e pesquisa (PIVETTA F; CARVALHO MAP, 2012). O estudo foi realizado entre os meses de março a abril de 2016, após ter sido aprovado pelo Comitê de Ética em Pesquisa da Escola Nacional de Saúde Pública Sérgio Arouca (ENSP/FIOCRUZ) sob o CAAE nㅇ 38554114.2.0000.5240.

Para capturar as experiências a partir das falas (memórias) dos partícipes sobre a realidade do trabalho e o das ações para compreender as práticas da EPS, foram realizados 3 grupos focais por categorias profissionais do cenário, sendo médicos (4), enfermeiros (5) e agentes comunitários de saúde (6), respeitando a disponibilidade dos mesmos. A proposta metodológica orientada pelo grupo focal justifica-se pela capacidade deste em promover a reflexão sobre o tema que suscitou emergir novas concepções, trazendo a possibilidade de análise e problematização de uma ideia em profundidade (BACKES DS, et al., 2011).

Nesse sentido, para sua realização seguiu-se a orientação de Patton M (2002) que pressupõe a formação de um pequeno grupo de pessoas para discutir um tópico específico. Definiu-se, assim, 03 (três) perguntas norteadoras aos grupos focais, a saber: Vocês, enquanto profissionais da ESF, identificam a EPS como espaço para a produção de saber in loco, no processo de trabalho? O que mudou nas práticas dos profissionais da ESF após a introdução da Educação Permanente em Saúde? Como a EPS influência nas práticas dos profissionais da ESF?

Os grupos focais foram gravados em áudio e um diário de campo permitiu a descrição de outros aspectos observados. Os dados foram transcritos e analisados à luz das principais referências e da PNEPS de $2004 \mathrm{e}$ 2007. Elencou-se, núcleos de sentido, que foram permeados pela comunicação, frequência e significância (BARDIN L, 2009).

Em seguida, realizou-se a codificação alfanumérica, em que MED, ENF e ACS, representaram, respectivamente, Médico, Enfermeiro e Agente Comunitário de Saúde, sendo que um número acompanhou e correspondeu a ordem de participação dos profissionais no estudo.

\section{RESULTADOS E DISCUSSÃO}

Os grupos focais possibilitaram a identificação da EPS enquanto espaço de produção de saberes para os profissionais da ESF, no entanto, estes são instituintes de mudanças na realidade do serviço. Nesta perspectiva as falas dos sujeitos explicitam a necessidade de aproximar as práticas educativas para dentro do contexto real dos profissionais a fim de alcançar por meio da EPS uma promoção de saberes no cotidiano do trabalho, esta aproximação entre educação e realidade também é discutida nas duas versões da PEPS. (BRASIL, 2004; BRASIL, 2009). 
"Eu acho que sim desde que esteja na realidade do profissional, não adianta numa área com hanseníase e tuberculose, você vir falar de síndrome não sei do quê, que não aparece muito aqui, eles precisam saber da realidade deles." (MED 2)

"Trazendo pra nossa realidade, às vezes é um assunto que é colocado muito e não chega à nossa realidade, então adequar o que a gente aprende trazendo pra dentro dos limites da nossa realidade." (ENF 3)

Para Ceccim RB e Feuerwerker LCM (2004) a EPS deve fazer uso de uma concepção construtivista de educação. Sarreta FO (2009) complementa, afirmando que, ela favorece a aprendizagem e potencializa a capacidade dos sujeitos de compreenderem o próprio trabalho a partir de suas vivências.

Sobre as práticas de EPS a Organização Mundial de Saúde (OMS) enfatiza a necessidade de desvendar o "saber como" e não o "saber tudo" (OMS, 2004), visando promoção de competências que gerem integração, apreensão de conhecimentos, trabalho em equipe e abordagem interdisciplinar (BRASIL, 2002).

A instituição como espaço de formação sob a lógica de gestão da EPS foi vista como relevante pelos profissionais, sendo que na ocasião do estudo o cenário possuía de acordo com o Regimento Interno espaço de encontro para EPS instituído todas as quintas-feiras, no horário de 13h00min às 17h00min.

No entanto, os participantes do estudo, iniciaram o processo de modificação solicitando a alteração do horário, visando envolvimento de mais atores e direcionamento das discussões face à realidade que envolve a prática dos profissionais de saúde.

"Acho que a gente poderia explorar mais, mais a gente não é questionado nesse sentido, quais são nossas demandas, quais são nossas necessidades. Os temas são estipulados e a gente vai (...)." (MED 3)

"As vezes existem coisas muito mais necessárias para serem tratadas e aí vem o pesquisador apresentar uma pesquisa, sobre uma coisa muito técnica, do doutorado que fez não sei lá aonde, então assim, as pessoas reclamam de ficar a tarde toda ali assistindo, sem nenhum interesse." (ENF 3)

"(...) como a maioria como tá acontecendo aí, é um trabalho que vem alguém apresentar que fez um Doutorado ou que fez um mestrado, alguma coisa assim." (ACS 2)

De acordo com a fala dos sujeitos as reuniões de EPS não conseguem atender as reais necessidades dos profissionais que têm a percepção da subutilização do espaço para a EPS, com pouco enfoque para questões que se aproximam da realidade das demandas cotidianas do trabalho, sendo utilizadas para atividades descontextualizadas, tais como a apresentação de resultados de pesquisas de doutores e mestres sem correlação direta com o trabalho desenvolvidos naquele cenário de cuidado em saúde.

A EPS deve estar imbricada com o que ocorre no cotidiano do trabalho e da vida, sendo o mundo do trabalho relacionado com o lugar ocupado pelos trabalhadores, que são atores fundamentais para a transformação da realidade.

Espaços como rodas de conversa e grupos de discussão devem ser implementados para se repensar o fazer saúde e discutir formas de cuidado engessadas. Desta forma, faz-se necessário que os profissionais de saúde tenham espaços de observação da sua realidade, para elencar os problemas, bem como para eleger possíveis estratégias educacionais de enfrentamento (STROSCHEIN KA; ZOCCHE DA, 2012).

Peduzzi M (1998) corroborando com os achados de Stroschein KA e Zocche DA (2012), afirma que os processos de trabalho que desenvolvem a melhoria do cuidado estão em algumas situações associadas ao trabalho coletivo de equipes multiprofissionais. A fala da participante enfermeira ganha tônus quando alinhada ao discurso dos autores citados acima:

"É também para qualificar o trabalho coletivo, porque a gente tem um contato maior com o nosso colega de trabalho, cada um trabalha de uma forma, a gente não uniformiza aqui dentro da unidade, mas é um falando da experiência do outro até 
ajuda e enriquece o trabalho do outro. Então eu acho que isso é uma coisa que só tem a acrescentar no profissional." (ENF 3)

O trabalho multiprofissional é inscrito como uma modalidade de trabalho coletivo que se configura na relação recíproca entre as intervenções técnicas e a interação dos agentes envolvidos. Nessa perspectiva, a comunicação seria um importante instrumento de negociação para a articulação das ações multiprofissionais (PEDUZZI M, 1998). A EPS conforme relato extraído dos grupos focais é capaz de promover saberes para os profissionais tanto do ponto de vista individual como no trabalho coletivo.

\begin{abstract}
"É um crescimento né, quando você acha interessante aquele assunto né, porque você pode pensar discutir, levar a quem é de direito aquele assunto, mas é uma forma de você pensar, de você interagir né, trabalhar junto." (ENF3)
\end{abstract}

Espera-se, que a EPS induza atuação transdisciplinar entre os profissionais de saúde, que alinhados numa mesma intencionalidade de cooperação buscarão um agir coletivo para o enfrentamento das fragilidades. Ferreira RC et al. (2009) identificou que a prática multiprofissional processa transformações no trabalho coletivo, pois no agir profissional em equipe os indivíduos acumulam a possibilidade de recompor suas práticas, construindo formas de intervenção ampliadas. A EPS como transformadora do processo de trabalho está orientada para uma constante melhoria da qualidade das ações de saúde (SALVADOR AS, et al., 2011), corroborando com a fala do ator acerca da reunião de equipe:

"A reunião de equipe é mais produtiva porque é a nossa realidade. Nós agentes comunitários é que trazemos os casos da comunidade para ser discutido na reunião, então é bem a nossa realidade é nossa prática." (ACS 3)

De acordo com Cruz MLS et al. (2008) a reunião de equipe deve ser uma possibilidade de encontro entre as pessoas para compartilhar o conhecimento (formal e informal) com o intuito de construir a partir disso uma estratégia de intervenção capaz de alterar uma dada realidade. A produção do conhecimento em saúde se caracteriza como um processo gerado no trabalho fundamentalmente participativo, já que resulta de em diferentes e complementares experiências entre a equipe de saúde e a comunidade. Nas falas abaixo é possível observar a importância da reunião de equipe.

"Teve uma época que as reuniões eram diferentes. Pegava o caso, fazia o genograma da família, ai cada equipe dava a sugestão, comentário, era uma coisa bem coletiva, bem mais interessante." (MED 2)

"A gente trazia demandas para a equipe, a gente tinha que escolher um caso problema pra ser discutido na reunião de equipe... Nesse modelo era muito bom... Ai cada pessoa dava a sugestão, comentários, era uma coisa bem coletiva, bem mai sinteressante." (MED 1)

A reunião de equipe é um importante espaço para produção de saberes. Nela as necessidades da equipe são discutidas e os profissionais conseguem interagir, aprendem junto, com as experiências de cada um, sendo um importante dispositivo para a estruturação, organização, informação, estabelecimento de diretrizes e espaço de tomada de decisões (GRANDO MK e DALL'AGNOL CM, 2010). Rojas FLL et al., (2019) considera que o construto educar e fazer saúde parte do ato no trabalho, na prática de saúde que ocorre continuamente com os sujeitos históricos e seus espaços.

O diálogo via discussão de caso ou através do projeto terapêutico singular (PTS) emerge como instrumento para a resolução dos problemas (BRASIL, 2007). O PTS contribui para o trabalho multiprofissional e integra o cuidado de forma a melhor atender as demandas, rompendo com a fragmentação e potencializando as ações em saúde (BRASIL, 2007). Já a reunião de equipe, no contexto que envolve o PTS, permite articulação de várias instâncias de saberes, promovendo planos de intervenção relacionados aos problemas relacionados às práticas de trabalho (LAURITO JAS, et al., 2018).

Para além da reunião formal de equipe, as reuniões de EPS enquanto espaço de produção do saber, foi percebido também nas falas que, segundo os profissionais, a EPS pode e deve ser vista integrada em todo 0 processo de trabalho, em que o ensino, serviço e a comunidade, o quadrilátero da saúde operem conjuntamente. 
"A comunidade, ali no dia a dia a gente vai aprendendo com eles no sentido de tentar ajudar e eles também aprendendo coma gente." (ACS 2)

"Bobo é quem acha que não aprende com a comunidade. Aprende muito. Todo dia tem uma coisa diferente que a gente vai aprendendo lá." (ACS 4)

Segundo os profissionais existem espaços onde ocorre a produção de aprendizagem que avançam modelos preconcebidos pela lógica de transferência do conhecimento. Esta particularidade de entender que os processos de aprendizagem acontecem a partir do processo de trabalho e da troca com os usuários, é uma vertente da Educação Permanente em Saúde, sendo a organização das equipes da Estratégia de Saúde da Família uma importante aliada para sua ocorrência da aproximação do profissional dos serviços com o cotidiano dos usuários.

No contexto da ESF para que tais atividades ocorram no processo de trabalho das equipes é importante que o profissional entenda seu contexto de trabalho e construa cadeias de produção de cuidado com os usuários pautados no entendimento das necessidades de saúde da comunidade e das famílias sob a sua responsabilidade, ou seja, ele precisa ter em mente o reproduzido pela fala do profissional participante do estudo e compreender que o "nosso próprio cotidiano é uma Educação Permanente né, por tudo o que a gente passa... que a gente coloca em prática tudo que a gente aprendeu e mais alguma coisa que a gente aprende com a prática né, a vivência mesmo do trabalho." (ENF 2)

É ser sensível, capturando o que ocorre no território vivo (do fazer, do saber, do sentir e do conviver) para compreender a subjetividade das famílias e dos usuários sob a sua responsabilidade como um fator importante para a produção de cuidado que esteja contextualizada com a realidade do usuário e da comunidade, para ser algo dinâmico que mude de acordo com as experiências de cada um e, que é afetada pelos valores e a cultura produzida socialmente. Partindo deste princípio, as práticas de trabalho vão além do uso de conhecimentos prescritivos, avançando para a produção do saber que necessita da integração entre o conhecimento teórico e as diferentes realidades identificadas, como observado abaixo.

“(...) não é EPS só quando tem reunião de equipe ou na quinta-feira a tarde quando se reúnem, mas sim a partir de um problema quando se reúnem profissionais ao redor daquele problema, para ter uma reflexão, ali é há uma produção de conhecimento (...)." (ENF 4)

Nesse contexto, no cotidiano do serviço deve ser estimulada a interação entre conhecimento teórico e a realidade vivenciada. Quintana PB et al. (1994) defendem que fica mais evidente a predominância do valor do atributo conhecimento em detrimento do exercício de análise das condições concretas do processo do trabalho. No que, Stroschein KA e Zocche DA (2012), salientam que quando a reflexão das práticas do trabalho em equipe nos serviços de saúde permanece na condição de segundo plano ou nem acontece a EPS não é contemplada.

O "comodismo" emergiu através da fala da participante dos grupos focais, sendo que este afeta as rotinas de trabalho e geram dificuldade para se inventar e reinventar novos processos, mais democráticos e participativos, "a gente involuntariamente sofre um processo de comodismo na rotina de trabalho e mudar causa medo [...], a questão da mudança a gente acaba tendo uma primeira reação involuntária de rejeição pelo próprio comodismo." (ENF 5)

Durante o diálogo, levantou-se a hipótese de que as mudanças nas estruturas do trabalho são complexas de ser realizadas porque envolvem toda uma modificação na forma de como o profissional e as instituições se organizam, então "[...] fica na tensão entre aquele modo de fazer que é enrijecido e é cristalizado mesmo de todo o ser humano como a capacidade de refletir sobre o seu próprio ato que é a educação permanente e você trazer aquilo que você vê a partir de um problema e estar sensibilizado a mudança.” (ENF 1)

A PNEPS reconhece que as rotinas de trabalho impedem a mudança ao inserir regras imutáveis, vistas como práticas consolidadas no tempo nas propostas de trabalho das instituições de saúde (BRASIL, 2009). Assim, a EPS deve estar comprometida com as reais necessidades dos serviços de saúde e com a transformação das práticas profissionais, com foco no desenvolvimento de competências. Assim, recomendase análise constante dos profissionais e da instituição, no que tange, aos processos de trabalho, visando identificar as pactuações de mudanças existentes e as possíveis propostas de intervenção. 


\section{CONCLUSÃO}

Constatou-se a presença de muitas tensões na EPS que influenciaram na sua configuração. No cenário, a EPS, poderia ser pensada sob duas vertentes, uma com foco nas questões que envolvem a gestão, e outra nas necessidades de desenvolvimento de competências da equipe multiprofissional. Os processos educativos mostraram-se capazes de contribuir para maior integração entre os profissionais, pelo fato de abrirem canais de comunicação para maior circulação do conhecimento. Observou-se fragilidades na formação do profissional ACS, exigindo atenção quanto a sua formação técnica. As reuniões de equipe emergiram como relevantes espaços de troca de saberes e produção da EPS. Já, a discussão de casos e o PTS, como definidoras de novos processos de trabalho.

\section{REFERÊNCIAS}

1. BRASIL. Ministério da Saúde. As Cartas da Promocão da Saúde. Brasília: Ministério da Saúde, 2002.

2. BRASIL. Ministério da Saúde. Política de educação e desenvolvimento para o SUS: caminhos para a educação permanente em saúde: pólos de educação permanente em saúde. Brasília: Ministério da Saúde, 2004.

3. BRASIL. Conselho Nacional de Secretảrios de Saúde. Atenção Primária e Promoção da Saúde. Brasília: CONASS,

4. BRAṠIL. Ministério da Saúde. Política Nacional de Educação Permanente em Saúde. Brasília: Ministério da Saúde, 2009.

5. BACKES DS, et al. Grupo focal como técnica de coleta e análise de dados em pesquisas qualitativas. O mundo da saúde,2011; 35 (4): 438-442.

6. BARDIN L. Análise de Conteúdo. Lisboa: Editora 70, 2010.

7. PIVETTA F, CARVALHO MAP. O território Integrado de atenção a saúde em Manguinhos: todos somos aprendizes! ENSP/FIOCRUZ, 2012

8. CAVALHEIRO MTP, GUUIMARÃES AL. Formação para o SUS e os desafios da integração ensino serviço. Caderno FNEPAS, 2011; 1: 19-27.

9. CECCIMN RB. Educação Permanente em Saúde: desafio ambicioso e necessário. Interface - Comunic, Saúde, Educ, 2005; 9 (16): 161-77.

10. CECCIM RB, FEUERWWERKER LCM. O Quadrilátero da Formação para a Área da Saúde: Ensino, Gestão, Atenção e Controle Social. PHYSIS: Rev. Saúde Coletiva, 2004; 14 (1): 41- 65.

11. CRUZ MLS, et al. Reunião de equipe: uma reflexão sobre sua importância enquanto estratégia diferencial na gestão coletiva no Programa de Saúde da Família (PSF). Psic. Rev. São Paulo, 2008;17 (1), nº 2: 161-183.

12. CSE MANGUINHOS. Regimento Interno Centro de Saúde Escola Germano Sinval Farias. Rio de Janeiro, 2014.

13. FARIA HP, et al. Modelo assistencial e atenção básica à saúde. 2ª ed. Belo Horizonte: Nescon/UFMG, 2010.

14. FERREIRẢ RC, et al. Trabalho em equipe multiprofissional: a perspectiva dos residentes médicos em saúde da família. Ciência \& Saúde Coletiva, 2009; 14 (1): 1421-1428.

15. GARCIA RM, BAPTISTA R. Educação a distância para a qualificação dos profissionais do SUS: perspectivas e desafios. Rev Baiana de Saúde Pública, 2007; 31(1): 70-78.

16. GOMES KO, et al. Avaliação do impacto do Programa Saúde da Família no perfil epidemiológico da população rural de Airões, município de Paula Cândido (MG), 1992- 2003. Ciência \& Saúde Coletiva, 2009; 14 (1): 1473-1482.

17. GRANDO MK, DALL'AGNOL CM. Desafios do processo grupal em reuniões de equipe da estratégia saúde da família. Esc Anna Nery (impr.), 2010; 14 (3): 504-510.

18. LAURITO JAS, et al. Proposta de instrumento para projeto terapêutico singular em saúde mental. Cadernos UNIFOA, Volta Redonda, 2018; 37, p. 115- 122.

19. NETO MMC. A Implantacáo da Unidade de Saúde da Família. Brasília: Ministério da Saúde, 2000.

20. PEDUZZI M. Equipe multiprofissional de saúde: a interface entre trabalho e interação (tese) - Universidade Estadual de Campinas, Campinas, 1998; $151 \mathrm{p}$.

21. PATTON M. Qualitative Research and Evaluation Methods, 3rd edn. Thousand Oaks, CA: Sage, 2002.

22. QUINTANA PB, et al. Educación Permanente, processo de trabajo y calidad de servicio en salud. In: HADDAD J.; ROSCHKE, M. A. L. C.; DAVINI, M. C. (org.) Educación Permanente de Personal de Salud. Washington: Organizacion Panamericana de la Salud. Série Desarrollo de Recursos Humanos no100, 1994: p. 34-60.

23. REZENDE MA. Articulação educação-saúde (AES) no processo de formulaccão das políticas nacionais voltadas para a formacão de nível superior dos profissionais de saúde. Rio de Janeiro: ENSP, 2013.

24. ROJAS FLL. Passos da Educação aos Caminhos da Aprendizagem em Saúde. Cuiabá: EDUFMT; 2018

25. ROJAS FLL, et al. Educação permanente em saúde: o repensar sobre a construção das práticas de saúde. J Health NPEPS, 2019; 4(2):310-330.

26. ROSA WAG, LABATE RC. Programa saúde da família: a construção de um novo modelo de assistência. Rev. LatinoAm. Enfermagem, 2005; 13(6): 1027-1034.

27. SALVADOR AS, et al. Construindo a Multiprofissionalidade: um Olhar sobre a Residência Multiprofissional em Saúde da Família e Comunidade. Revista Brasileira de Ciências da Saúde, 2011; 15 (3): 329-338.

28. SARRETA FO. Educação permanente em saúde para os trabalhadores do SUS. São Paulo: UNESP, 2009; 252p.

29. SOBRAL FAF. A universidade e o novo modo de produção do conhecimento. Caderno CRH: Centro de Recursos Humanos da UFBA, Salvador, 2001; 34: 265-275.

30. SMSDC. Secretaria Municipal de Saúde e Defesa Civil. Planejamento Estratégico da Prefeitura do Rio de Janeiro 2009 - 2012. RIO DE JANEIRO, 2008.

31. SMSSDC. Superintendência de Atenção Primária. Guia de Referência Rápida. Carteira de Serviços: Relação de serviços prestados na Atenção Primária à Saúde / Secretaria Municipal de Saúde e Defesa Civil. Superintendência de Atenção Primária. - Rio de Janeiro: SMSDC, 2011.

32. STROSCHEIN KA, ZOCCHE DA. A Educação permanente nos servicos de saúde: um estudo sobre as experiências realizadas no Brasil. Trabalho, Educação e Saúde, Rio de Janeiro, 2012; 9 (3): 505-519. 